\title{
Another Approach to Juhl's Conformally Covariant Differential Operators from $S^{n}$ to $S^{n-1}$
}

Jean-Louis CLERC

Institut Elie Cartan de Lorraine, Université de Lorraine, France

E-mail: jean-louis.clerc@univ-lorraine.fr

Received December 07, 2016, in final form April 11, 2017; Published online April 19, 2017

https://doi.org/10.3842/SIGMA.2017.026

\begin{abstract}
A family $\left(\mathbf{D}_{\lambda}\right)_{\lambda \in \mathbb{C}}$ of differential operators on the sphere $S^{n}$ is constructed. The operators are conformally covariant for the action of the subgroup of conformal transformations of $S^{n}$ which preserve the smaller sphere $S^{n-1} \subset S^{n}$. The family of conformally covariant differential operators from $S^{n}$ to $S^{n-1}$ introduced by A. Juhl is obtained by composing these operators on $S^{n}$ and taking restrictions to $S^{n-1}$.
\end{abstract}

Key words: conformally covariant differential operators; Juhl's covariant differential operators

2010 Mathematics Subject Classification: 58J70; 43A85

\section{Introduction}

Let $S=S^{n}$ be the $n$-dimensional sphere in $\mathbb{R}^{n+1}$ and let $G=\mathrm{SO}_{0}(1, n+1)$ be (the neutral component of) the group of conformal transformations of $S$. Let $S^{\prime} \simeq S^{n-1}$ be the subspace of points of $S$ with vanishing last coordinate $\left(x_{n}=0\right.$ in our notation) and let $G^{\prime} \simeq \mathrm{SO}_{0}(1, n)$ be the conformal group of $S^{\prime}$, viewed as the subgroup of $G$ which stabilizes $S^{\prime}$. Let $\left(\pi_{\lambda}\right)_{\lambda \in \mathbb{C}}$ be the scalar principal series of representations of $G$ acting on $C^{\infty}(S)$. Denote by $\pi_{\lambda \mid G^{\prime}}$ its restriction to $G^{\prime}$. Let $\left(\pi_{\mu}^{\prime}\right)_{\mu \in \mathbb{C}}$ be the scalar principal series of $G^{\prime}$ acting on $C^{\infty}\left(S^{\prime}\right)$.

In [6] A. Juhl has constructed a family $\mathcal{D}_{N}(\lambda)_{\lambda \in \mathbb{C}, N \in \mathbb{N}}$ of differential operators from $C^{\infty}(S)$ into $C^{\infty}\left(S^{\prime}\right)$, which are intertwining operators between $\pi_{\lambda \mid G^{\prime}}$ and $\pi_{\lambda+N}^{\prime}{ }^{1}$ Later, these operators were obtained by T. Kobayashi and B. Speh in [11] as residues of a meromorphic family of symmetry breaking operators associated to the restriction problem for the pair $\left(G, G^{\prime}\right)$. A third point of view was proposed by T. Kobayashi and M. Pevzner in $[9,10]$, based on the $F$-method. Similar operators were recently constructed for differential forms on spheres $[4,8]$.

The new approach to Juhl's operators which I present in this article follows a method that I used for similar problems, in the context of the restriction problem for a pair $\left(G \times G, G^{\prime}\right)$ where $G^{\prime}=G$ embedded diagonally in $G \times G$. I was influenced by a reminiscence of the $\Omega$-process which yields both the transvectants and the Rankin-Cohen brackets. These operators may be viewed as covariant bi-differential operators for the group $\operatorname{SL}(2, \mathbb{R})$, or symmetry breaking differential operators from $\mathrm{SL}(2, \mathbb{R}) \times \mathrm{SL}(2, \mathbb{R})$ to its diagonal subgroup. For a presentation of these classical results see Section 5 of [2] for a quick overview or [12] for a thorough exposition of the transvectants.

The new method was introduced in a collaboration with R. Beckmann for the conformal group of the sphere (see [1]) and the scalar principal series, then for $G=\operatorname{SL}(2 n, \mathbb{R})$ and the degenerate principal series acting on the Grasmmannian $\operatorname{Gr}(n, 2 n ; \mathbb{R})$ (see [2]).

The first step of the method, for the present case, is to introduce the multiplication by $x_{n}$, viewed as an operator $M$ on $C^{\infty}(S)$. The operator $M$ is a "universal" $G^{\prime}$-intertwining operator,

\footnotetext{
${ }^{1}$ Our $\lambda$ corresponds to $-\lambda$ in Juhl's notation.
} 
in the sense that, for any $\lambda \in \mathbb{C}$, the operator $M$ intertwines $\pi_{\lambda \mid G^{\prime}}$ and $\pi_{\lambda-1 \mid G^{\prime}}$. Next recall the family of Knapp-Stein operators $\left(I_{\lambda}\right)_{\lambda \in \mathbb{C}}$ which are $G$-intertwining operators with respect to $\left(\pi_{\lambda}, \pi_{n-\lambda}\right)$. The operator ${ }^{2}$

$$
\mathbf{D}_{\lambda}=I_{n-\lambda-1} \circ M \circ I_{\lambda}
$$

obtained by twisting $M$ by the appropriate Knapp-Stein intertwining operators is clearly an intertwining operator with respect to $\left(\pi_{\lambda \mid G^{\prime}}, \pi_{\lambda+1 \mid G^{\prime}}\right)$. Our main result (see Theorem 3.2) is that $\mathbf{D}_{\lambda}$ is a differential operator. The proof is obtained in the non compact realization of the principal series (passing from $S^{n}$ to $\mathbb{R}^{n}$ by a conformal map) and uses Euclidean Fourier transform.

The construction of conformally covariant differential operators from $S^{n}$ to $S^{n-1}$ is now easy. For $N$ a non-negative integer, consider

$$
\mathbf{D}_{N, \lambda}=\mathbf{D}_{\lambda+N-1} \circ \cdots \circ \mathbf{D}_{\lambda+1} \circ \mathbf{D}_{\lambda} \quad \text { or } \quad \mathbb{D}_{N, \lambda}=I_{n-\lambda-N} \circ M^{N} \circ I_{\lambda} .
$$

The two families of differential operators on $S$ (which coincide up to a meromorphic function of $\lambda)$ are covariant with respect to $\left(\pi_{\lambda \mid G^{\prime}}, \pi_{\lambda+N \mid G^{\prime}}\right)$. Finally, let

$$
\mathbf{D}_{N}(\lambda)=\operatorname{res} \circ \mathbf{D}_{N, \lambda}
$$

where res is the restriction map from $C^{\infty}(S)$ to $C^{\infty}\left(S^{\prime}\right)$. The operator $\mathbf{D}_{N}(\lambda)$ is a differential operator from $S$ to $S^{\prime}$ which is covariant with respect to $\left(\pi_{\lambda \mid G^{\prime}}, \pi_{\lambda+N}^{\prime}\right)$. The family $\mathbf{D}_{N}(\lambda)_{\lambda \in \mathbb{C}, N \in \mathbb{N}}$ essentially coincides with Juhl's family.

The operator $\mathbf{D}_{\lambda}$ has a simple expression in the non compact picture, see (4.6). It is tempting to find a more direct approach to this operator. This is achieved in the last section, by using yet another realization of the principal series, sometimes called the ambient space realization. The way the operator is constructed is much simpler, and it is then easy to determine its expression in the non compact picture (recovering the expression of $\mathbf{D}_{\lambda}$ on $\mathbb{R}^{n}$, see Proposition 7.8), but also in the compact realization (see Proposition 7.9), that is to say as a $G^{\prime}$-conformally covariant differential operator on $S$. Some generalization of these formulæ in the realm of conformal geometry on a Riemannian manifold seems plausible.

\section{The principal series of $\mathrm{SO}_{0}(1, n+1)$ and the Knapp-Stein intertwining operators}

Let $E$ be a Euclidean space of dimension $n+1$, and choose an orthonormal basis $\left\{e_{0}, e_{1}, \ldots, e_{n}\right\}$. Let $S=S^{n}$ be the unit sphere of $E$, i.e.,

$$
S=\left\{x=\left(x_{0}, x_{1}, \ldots, x_{n}\right), x_{0}^{2}+x_{1}^{2}+\cdots+x_{n}^{2}=1\right\} .
$$

Let $\mathbf{E}$ be the vector space $\mathbb{R} \oplus E$, with the Lorentzian quadratic form

$$
Q(\mathbf{x})=[(t, x),(t, x)]=t^{2}-|x|^{2} \quad \text { for } \quad \mathbf{x}=(t, x), \quad t \in \mathbb{R}, \quad x \in E .
$$

For $\mathbf{x}=(t, x) \in \mathbf{E}$, we let

$$
t(\mathbf{x})=t, \quad \mathbf{x}_{E}=x .
$$

The space of isotropic lines $\mathcal{S}$ in $\mathbf{E}$ can be identified with $S$ by the map

$$
S \ni x \longmapsto d_{x}=\mathbb{R}(1, x) \in \mathcal{S}, \quad \mathcal{S} \ni d \longmapsto d \cap\{t=1\} .
$$

\footnotetext{
${ }^{2}$ For technical reasons, a normalizing factor is introduced, see (3.4).
} 
Let $G=\mathrm{SO}_{0}(1, n+1)$ be the connected component of the group of isometries of $\mathbf{E}$. Then $G$ acts on $\mathcal{S}$ and this action can be transferred to an action on $S$. More explicitly, if $x=$ $\left(x_{0}, x_{1}, \ldots, x_{n}\right) \in S$, and $g \in G$, observe that $t(g(1, x))>0$ and define $g(x) \in S$ by

$$
(1, g(x))=t(g \cdot(1, x))^{-1} g \cdot(1, x) .
$$

Set, for $g \in G$ and $x \in S$

$$
\kappa(g, x)=t(g \cdot(1, x))^{-1} .
$$

Clearly $\kappa(g, x)$ is a smooth, strictly positive function on $G \times S$. Moreover $\kappa(g, x)$ satisfies the cocycle property: for any $g_{1}, g_{2}$ and any $x \in S$,

$$
\kappa\left(g_{1} g_{2}, x\right)=\kappa\left(g_{1}, g_{2}(x)\right) \kappa\left(g_{2}, x\right) .
$$

This action of $G$ on $S$ is known to be conformal. For $g \in G, x \in S$ and $\xi$ an arbitrary tangent vector to $S$ at $x$

$$
|D g(x) \xi|=\kappa(g, x)|\xi|
$$

and hence $\kappa(g, x)$ is the conformal factor of $g$ at $x$.

Associated to the action of $G$ on $S$ there is a family of representations on $C^{\infty}(S)$, which, from the point of view of harmonic analysis is the scalar principal series of $G$. For $\lambda \in \mathbb{C}, g \in G$ and $f \in C^{\infty}(S)$, let

$$
\pi_{\lambda}(g) f(x)=\kappa\left(g^{-1}, x\right)^{\lambda} f\left(g^{-1}(x)\right) .
$$

The formula defines a (smooth) representation $\pi_{\lambda}$ of $G$ on $C^{\infty}(S)$.

The Knapp-Stein intertwining operators are a major tool in harmonic analysis of $G$ (as of any semi-simple Lie group, see, e.g., [7]). For $\lambda \in \mathbb{C}$ and $f \in C^{\infty}(S)$, let

$$
I_{\lambda} f(x)=\frac{1}{\Gamma\left(\lambda-\frac{n}{2}\right)} \int_{S}|x-y|^{-2 n+2 \lambda} f(y) d y,
$$

where $d y$ stands for the Lebesgue measure on $S$ induced by the Euclidean structure. For $\operatorname{Re} \lambda>\frac{n}{2}$, this formula defines a continuous operator $I_{\lambda}$ on $C^{\infty}(S)$.

\section{Proposition 2.1.}

i) The definition (2.1) can be analytically continued in $\lambda$ to all of $\mathbb{C}$.

ii) The analytic continuation yields a holomorphic family of operators $I_{\lambda}$ on $C^{\infty}(S)$, which satisfy the intertwining relation

$$
\forall g \in G, \quad I_{\lambda} \circ \pi_{\lambda}(g)=\pi_{n-\lambda}(g) \circ I_{\lambda} .
$$

The following complementary result will be needed later.

Proposition 2.2. For any $\lambda \in \mathbb{C}$

$$
I_{\lambda} \circ I_{n-\lambda}=\frac{\pi^{n}}{\Gamma(\lambda) \Gamma(n-\lambda)} \mathrm{id} .
$$


The next result corresponds to reducibility points for the scalar principal series. Let $\mathcal{P}(S)$ be the space of restrictions to $S$ of polynomial functions on $E$, and for $k \in \mathbb{N}$, let $\mathcal{P}_{k}$ be the space of restrictions to $S$ of polynomials on $E$ of degree $\leq k$. Finally, let $\mathcal{P}_{k}^{\perp}$ be the subspace of $C^{\infty}(S)$ given by

$$
\mathcal{P}_{k}^{\perp}=\left\{f \in C^{\infty}(S), \int_{S} f(x) p(x) d x=0, \text { for any } p \in \mathcal{P}_{k}\right\} .
$$

\section{Proposition 2.3.}

i) Let $\lambda=n+k, k \in \mathbb{N}$. Then

$$
\operatorname{Im}\left(I_{n+k}\right)=\mathcal{P}_{k}, \quad \operatorname{Ker}\left(I_{n+k}\right)=\mathcal{P}_{k}^{\perp} .
$$

ii) Let $\lambda=-k, k \in \mathbb{N}$. Then

$$
\operatorname{Ker}\left(I_{-k}\right)=\mathcal{P}_{k}, \quad \operatorname{Im}\left(I_{-k}\right)=\mathcal{P}_{k}^{\perp} .
$$

\section{Construction of the family $\widetilde{\mathrm{D}}_{\lambda}, \lambda \in \mathbb{C}$}

Now let $E^{\prime}=\left\{x \in E, x_{n}=0\right\}$ and $S^{\prime}=S \cap E^{\prime}$. Then $S^{\prime}$ is an $(n-1)$-dimensional sphere. Let $G^{\prime}$ be the subgroup of elements of $G$ of the form

$$
g=\left(\begin{array}{cccc} 
& & & 0 \\
& g^{\prime} & & \vdots \\
& & & 0 \\
0 & \ldots & 0 & 1
\end{array}\right), \quad g^{\prime} \in \operatorname{SO}_{0}(1, n) .
$$

Clearly, $G^{\prime}$ is a subgroup of $G$, isomorphic to $\mathrm{SO}_{0}(1, n)$. Elements of $G^{\prime}$ preserve the hyperplane $\left\{x_{n}=0\right\}$ in $\mathbf{E}$ and hence the action of $G^{\prime}$ on $S$ preserves $S^{\prime}$.

For $x \in E$, write $x=\left(x^{\prime}, x_{n}\right)$, with $x^{\prime} \in \mathbb{R}^{n}$. For $g \in G^{\prime}$,

$$
g(1, x)=g\left(1, x^{\prime}, x_{n}\right)=\left(g^{\prime} \cdot\left(1, x^{\prime}\right), x_{n}\right) .
$$

If $x \in S$, the last equation can be rewritten as

$$
\kappa(g, x)^{-1}(1, g(x))=\left(g^{\prime} \cdot\left(1, x^{\prime}\right), x_{n}\right),
$$

so that

$$
g(x)_{n}=\kappa(g, x) x_{n} .
$$

In the sequel, the distinction between $g$ and $g^{\prime}$ in the notation is abandoned, the context providing the correct interpretation.

Let $M$ be the operator defined on $C^{\infty}(S)$ by

$$
M f(x)=x_{n} f(x), \quad f \in C^{\infty}(S) .
$$

Proposition 3.1. The operator $M$ satisfies

$$
\forall g \in G^{\prime} \quad M \circ \pi_{\lambda}(g)=\pi_{\lambda-1}(g) \circ M .
$$

Proof. This is an immediate consequence of (3.1). 
Next let $\mathbf{D}_{\lambda}$ be the operator on $C^{\infty}(S)$ defined by

$$
\mathbf{D}_{\lambda}=I_{n-\lambda-1} \circ M \circ I_{\lambda}
$$

which corresponds to the following diagram

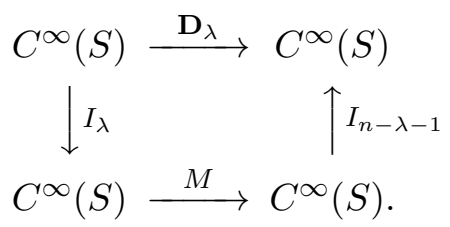

As a consequence of the intertwining property of the Knapp-Stein operators (2.2) and Proposition 3.1, $\mathbf{D}_{\lambda}$ satisfies for $g \in G^{\prime}$

$\mathbf{D}_{\lambda} \circ \pi_{\lambda}(g)=\pi_{\lambda+1}(g) \circ \mathbf{D}_{\lambda}$

Otherwise said, the operator $\mathbf{D}_{\lambda}$ is covariant with respect to $\left(\pi_{\lambda \mid G^{\prime}}, \pi_{\lambda+1 \mid G^{\prime}}\right)$.

Theorem 3.2. The operator $\mathbf{D}_{\lambda}$ is a differential operator on $S$.

The proof of Theorem 3.2 will be given at the end the next section.

Proposition 3.3. Let $\lambda \in(n+\mathbb{N}) \cup(-1-\mathbb{N})$. Then $\mathbf{D}_{\lambda}=0$.

Proof. Let first $\lambda=n+k$ for some $k \in \mathbb{N}$. Then $I_{\lambda}=I_{n+k}$, and by (2.4) $\operatorname{Im}\left(I_{\lambda}\right)=\mathcal{P}_{k}$. Next $\operatorname{Im}\left(M \circ I_{\lambda}\right) \subset \mathcal{P}_{k+1}$. Now $I_{n-\lambda-1}=I_{-k-1}$ and using $(2.5), I_{n-\lambda-1} \circ M \circ I_{\lambda}=0$.

Now let $\lambda=-k$, with $k \geq 1$. Then $I_{\lambda}=I_{-k}$ and by $(2.5), \operatorname{Im}\left(I_{\lambda}\right)=\mathcal{P}_{k}^{\perp}$. $\operatorname{Next} \operatorname{Im}\left(M \circ I_{\lambda}\right) \subset$ $\mathcal{P}_{1} \mathcal{P}_{k}^{\perp} \subset \mathcal{P}_{k-1}^{\perp}$. Now $I_{n-\lambda-1}=I_{n+k-1}$ which using (2.4) implies $I_{n-\lambda-1} \circ M \circ I_{\lambda}=0$.

To compensate for these zeroes of $\mathbf{D}_{\lambda}$, introduce

$$
\widetilde{\mathbf{D}}_{\lambda}=\Gamma(\lambda+1) \Gamma(n-\lambda) \mathbf{D}_{\lambda}
$$

for $\lambda \notin(n+\mathbb{N}) \cup(-1-\mathbb{N})$ and extend continuously to all of $\mathbb{C}$ to get a holomorphic family $\left(\widetilde{\mathbf{D}}_{\lambda}\right)_{\lambda \in \mathbb{C}}$ of differential operators on $S$ covariant with respect to $\left(\pi_{\lambda \mid G^{\prime}}, \pi_{\lambda+1 \mid G^{\prime}}\right)$.

\section{The expression of $\widetilde{\mathrm{D}}_{\lambda}$ in the non-compact picture}

Consider the point $\mathbf{- 1}=(-1,0, \ldots, 0) \in S$. The stereographic projection with source at $\mathbf{- 1}$ provides a diffeomorphism from $S \backslash\{-\mathbf{1}\}$ onto the hyperplane $\left\{x_{n}=1\right\}$. The inverse map (up to a scaling by a factor 2) $c: \mathbb{R}^{n} \longrightarrow S$ is given by

$$
c(\xi)=\left(\begin{array}{c}
\frac{1-|\xi|^{2}}{1+|\xi|^{2}} \\
\frac{2 \xi_{1}}{1+|\xi|^{2}} \\
\vdots \\
\frac{2 \xi_{n}}{1+|\xi|^{2}}
\end{array}\right)
$$

When using this local chart on $S$, we refer to the non-compact picture, as a reference to semisimple harmonic analysis. 
Geometric considerations (or an elementary computation) show that, for $\xi, \eta \in \mathbb{R}^{n}$

$$
|c(\xi)-c(\eta)|^{2}=\kappa(c, \xi)|\xi-\eta|^{2} \kappa(c, \eta),
$$

where, for $\xi \in \mathbb{R}^{n}$, we set

$$
\kappa(c, \xi)=2\left(1+|\xi|^{2}\right)^{-1} .
$$

There is an infinitesimal version of this result, namely

$$
|D c(\xi) \eta|=\kappa(c, \xi)|\eta|
$$

for $\xi, \eta \in \mathbb{R}^{n}$. This last statement shows that $c$ is conformal from $\mathbb{R}^{n}$ with its standard Euclidean structure into $S$.

The action of $g$ on $S$ can be transferred as a (rational) action of $G$ on $\mathbb{R}^{n}$, namely $c^{-1} \circ g \circ c$. For notational convenience, we still denote this action on $\mathbb{R}^{n}$ by $(g, \xi) \longmapsto g(\xi), g \in G, \xi \in \mathbb{R}^{n}$. As the map $c$ is conformal, the transferred action of $G$ on $\mathbb{R}^{n}$ is still conformal. For $g \in G$ defined at $\xi \in \mathbb{R}^{n}$, we let $\kappa(g, \xi)$ be the corresponding conformal factor of $g$ at $\xi$.

Let $\lambda \in \mathbb{C}$. For $f \in C^{\infty}(S)$ let $C_{\lambda}(f)$ be defined by

$$
C_{\lambda}(f)(\xi)=\kappa(c, \xi)^{\lambda} f(c(\xi)), \quad \xi \in \mathbb{R}^{n}
$$

and let $\mathcal{H}_{\lambda}$ be the image of $C_{\lambda}$. It is easily proved that

$$
\mathcal{S}\left(\mathbb{R}^{n}\right) \subset \mathcal{H}_{\lambda} \subset \mathcal{S}^{\prime}\left(\mathbb{R}^{n}\right),
$$

where $\mathcal{S}\left(\mathbb{R}^{n}\right)$ stands for the Schwartz space on $\mathbb{R}^{n}$ and $\mathcal{S}^{\prime}\left(\mathbb{R}^{n}\right)$ for its dual, the space of tempered distributions.

The representation $\pi_{\lambda}$ can be transferred in the non-compact model, using $C_{\lambda}$ as intertwining map, i.e., set

$$
\rho_{\lambda}(g)=C_{\lambda} \circ \pi_{\lambda}(g) \circ C_{\lambda}^{-1} .
$$

Using the cocycle property of $\kappa, \rho_{\lambda}$ can be realized as

$$
\rho_{\lambda}(g) f(\xi)=\kappa\left(g^{-1}, \xi\right)^{\lambda} f\left(g^{-1}(\xi)\right),
$$

where $f \in \mathcal{H}_{\lambda}$ and $g \in G$.

Similarly, the Knapp-Stein operators can be transferred to the non-compact picture. For $s \in \mathbb{C}$, consider the expression

$$
h_{s}(\xi)=\frac{1}{\Gamma\left(\frac{n}{2}+\frac{s}{2}\right)}|\xi|^{s}, \quad \xi \in \mathbb{R}^{n} .
$$

For $\operatorname{Re}(s)>-n, h_{s}$ is locally summable with moderate growth at infinity, hence defines a tempered distribution. The $\left(\mathcal{S}^{\prime}\left(\mathbb{R}^{n}\right)\right.$-valued) function $s \mapsto h_{s}$ can be extended by analytic continuation to $\mathbb{C}$ and the $\Gamma$ factor in the definition of $h_{s}$ is so chosen that it extends as an entire function with values in $\mathcal{S}^{\prime}\left(\mathbb{R}^{n}\right)$ (for more details see, e.g., [5]).

For $\lambda \in \mathbb{C}$, the Knapp-Stein operator $J_{\lambda}$ is given by

$$
J_{\lambda} f=h_{-2 n+2 \lambda} \star f,
$$

or more concretely

$$
J_{\lambda} f(\xi)=\frac{1}{\Gamma\left(\lambda-\frac{n}{2}\right)} \int_{\mathbb{R}^{n}}|\xi-\eta|^{-2 n+2 \lambda} f(\eta) d \eta .
$$

As for any $s \in \mathbb{C} h_{s}$ is a tempered distribution, $J_{\lambda}$ maps $\mathcal{S}\left(\mathbb{R}^{n}\right)$ into $\mathcal{S}^{\prime}\left(\mathbb{R}^{n}\right)$. 
Proposition 4.1. Let $\lambda \in \mathbb{C}$. Then for $f \in \mathcal{S}\left(\mathbb{R}^{n}\right)$

$$
J_{\lambda} f=\left(C_{n-\lambda} \circ I_{\lambda} \circ C_{\lambda}^{-1}\right) f .
$$

Proof. As $\mathcal{S}\left(\mathbb{R}^{n}\right) \subset \mathcal{H}_{\lambda} \subset \mathcal{S}^{\prime}\left(\mathbb{R}^{n}\right)$, both sides are well-defined and belong to $\mathcal{S}^{\prime}\left(\mathbb{R}^{n}\right)$. For $\operatorname{Re} \lambda>\frac{n}{2}$, both sides are given by convergent integrals, and the equality is proved by a change of variable. The general case follows by analytic continuation. The intertwining property of the Knapp-Stein operators can be formulated in the following way.

Proposition 4.2. Let $f \in C_{c}^{\infty}(S)$ and let $g \in G$ such that $g^{-1}$ is defined on $\operatorname{Supp}(f)$. Then

$$
J_{\lambda}\left(\rho_{\lambda}(g) f\right)=\rho_{n-\lambda}(g)\left(J_{\lambda} f\right),
$$

where the two sides of the equation are viewed as tempered distributions on $\mathbb{R}^{n}$.

Proof. The condition implies that both $f$ and $\rho_{\lambda}(g) f$ are contained in $\mathcal{S}\left(\mathbb{R}^{n}\right)$. Hence

$$
\begin{aligned}
J_{\lambda}\left(\rho_{\lambda}(g) f\right) & =\left(C_{n-\lambda} \circ I_{\lambda} \circ C_{\lambda}^{-1}\right)\left(\rho_{\lambda}(g) f\right) \\
& =\left(C_{n-\lambda} \circ I_{\lambda}\right)\left(\pi_{\lambda}(g) C_{\lambda}^{-1} f\right)=\left(C_{n-\lambda} \circ \pi_{n-\lambda}(g)\right) \circ\left(I_{\lambda} \circ C_{\lambda}^{-1}\right) f \\
& =\rho_{n-\lambda}(g) \circ\left(C_{n-\lambda} \circ I_{\lambda} \circ C_{\lambda}^{-1}\right) f=\rho_{n-\lambda}(g)\left(J_{\lambda} f\right) .
\end{aligned}
$$

The following formulæ will be needed in the sequel

$$
\begin{aligned}
|\xi|^{2} h_{s}(\xi) & =\frac{n+s}{2} h_{s+2}(\xi), \\
\frac{\partial}{\partial \xi_{n}} h_{s}(\xi) & =\frac{2 s}{n+s-2} \xi_{n} h_{s-2}(\xi),
\end{aligned}
$$

where at $s=-n+2$, the last formula has to be understood by analytic continuation.

As the pole of the stereographic projection has been chosen in $S^{\prime}$, the map $c$ maps the hyperplane $\left\{\xi_{n}=0\right\}$ into $S^{\prime}$. It allows to transfer the map $M$ to the non-compact picture.

Lemma 4.3. Let $g \in G^{\prime}$ be defined at $\xi \in \mathbb{R}^{n}$. Then

$$
g(\xi)_{n}=\kappa(g, \xi) \xi_{n}
$$

Proof. Let $\xi \in \mathbb{R}^{n}$ and let $x=c(\xi) \in S \backslash\{\mathbf{- 1}\}$. Then

$$
c(\xi)_{n}=\kappa(c, \xi) \xi_{n}, \quad g(x)_{n}=\kappa(g, x) x_{n}, \quad c^{-1}(x)=\kappa\left(c^{-1}, x\right) x_{n}
$$

the first equality by (4.1), the second by (3.1), and the third also by (4.1). As $\kappa$ satisfies a cocycle relation, we get

$$
\left(\left(c^{-1} \circ g \circ c\right)(\xi)\right)_{n}=\kappa\left(c^{-1} \circ g \circ c, \xi\right) \xi_{n},
$$

which gives (4.4).

Lemma 4.4. Let $\lambda \in \mathbb{C}$ and $f \in C^{\infty}(S)$. Then

$$
C_{\lambda-1}(M f)(\xi)=\xi_{n} C_{\lambda}(f)(\xi), \quad \xi \in \mathbb{R}^{n} .
$$

Proof. Let $\xi=\left(\xi^{\prime}, \xi_{n}\right)$. By $(4.1), c(\xi)_{n}=\kappa(c, \xi) \xi_{n}$, so that

$$
C_{\lambda-1}(M f)(\xi)=\kappa(c, \xi)^{\lambda-1} M f(c(\xi))=\kappa(c, \xi)^{\lambda} \xi_{n} f(c(\xi))=\xi_{n} C_{\lambda}(f)(\xi) .
$$


Abusing notation, $M$ will be used for the operator (on $C^{\infty}\left(\mathbb{R}^{n}\right)$ say) of multiplication by $\xi_{n}$. The operator $M$ maps $\mathcal{S}\left(\mathbb{R}^{n}\right)$ (resp. $\mathcal{S}^{\prime}\left(\mathbb{R}^{n}\right)$ ) into $\mathcal{S}\left(\mathbb{R}^{n}\right)$ (resp. $\mathcal{S}^{\prime}\left(\mathbb{R}^{n}\right)$ ), and for any $\lambda \in \mathbb{C}$, the operator $M$ maps $\mathcal{H}_{\lambda}$ into $\mathcal{H}_{\lambda-1}$ (Lemma 4.4).

Proposition 4.5. Let $\lambda \in \mathbb{C}$. The operator $M: \mathcal{H}_{\lambda} \longrightarrow \mathcal{H}_{\lambda-1}$ satisfies

$$
\forall g \in G^{\prime}, \quad M \circ \rho_{\lambda}(g)=\rho_{\lambda-1}(g) \circ M .
$$

Otherwise said, the operator $M$ intertwines the representations $\pi_{\lambda \mid G^{\prime}}$ and $\pi_{\lambda-1 \mid G^{\prime}}$.

Proof. Let $f \in \mathcal{H}_{\lambda}$. Then

$$
\begin{aligned}
\left(M \circ \rho_{\lambda}(g)\right) f(\xi) & =\xi_{n} \kappa\left(g^{-1}, \xi\right)^{\lambda} f\left(g^{-1}(\xi)\right)=\kappa\left(g^{-1}, \xi\right)^{\lambda-1}\left(g^{-1}(\xi)\right)_{n} f\left(g^{-1}(\xi)\right) \\
& =\rho_{\lambda-1}(g)(M f)\left(g^{-1}(\xi)\right)
\end{aligned}
$$

and the statement follows.

Having introduced the non-compact version of the main ingredients, we observe that the Knapp-Stein operators are convolution operators, whereas $M$ is the multiplication by an elementary polynomial. So the Fourier transform is well-fitted for computations in this context. Define the Fourier transform on $\mathbb{R}^{n}$ as usual by

$$
\widehat{f}(\eta)=\int_{\mathbb{R}^{n}} e^{i\langle\eta, \xi\rangle} f(\xi) d \xi
$$

initially for functions in $\mathcal{S}\left(\mathbb{R}^{n}\right)$ and extend by duality to $\mathcal{S}^{\prime}\left(\mathbb{R}^{n}\right)$.

The Fourier transform of $h_{s}$ is given by

$$
\widehat{h}_{s}=2^{n+s} \pi^{\frac{n}{2}} h_{-n-s} .
$$

For this result see, e.g., [5].

Thanks to the above observations, it is possible to define the composition $M \circ J_{\lambda}$ as an operator from $\mathcal{S}\left(\mathbb{R}^{n}\right)$ into $\mathcal{S}^{\prime}\left(\mathbb{R}^{n}\right)$.

Lemma 4.6. For $f \in \mathcal{S}\left(\mathbb{R}^{n}\right)$,

$$
\left(\left(M \circ J_{\lambda}\right) \hat{f)}(\eta)=-i \pi^{\frac{n}{2}} 2^{-n+2 \lambda}\left(h_{n-2 \lambda}(\eta) \frac{\partial \widehat{f}}{\partial \eta_{n}}(\eta)+\frac{n-2 \lambda}{n-\lambda-1} \eta_{n} h_{n-2-2 \lambda}(\eta) \widehat{f}(\eta)\right) .\right.
$$

Proof. As observed earlier, the Knapp-Stein operator $J_{\lambda}$ is a convolution operator on $\mathbb{R}^{n}$, so that

$$
\left(J_{\lambda} f\right)^{\widehat{ }}(\eta)=\widehat{h}_{-2 n+2 \lambda}(\eta) \widehat{f}(\eta)=2^{-n+2 \lambda} \pi^{\frac{n}{2}} h_{n-2 \lambda}(\eta) \widehat{f}(\eta) .
$$

Next, for any distribution $\varphi \in \mathcal{S}^{\prime}\left(\mathbb{R}^{n}\right)$

$$
\widehat{M \varphi}=-i \frac{\partial}{\partial \eta_{n}} \widehat{\varphi}
$$

and (4.5) follows, using (4.3).

The composition $J_{n-\lambda-1} \circ M \circ J_{\lambda}$ is not well-defined on $\mathcal{S}\left(\mathbb{R}^{n}\right)$. However, a formal computation (using again Fourier transforms) can be made and leads to a differential operator, which is at the origin of the definition (4.6) below. In order to give a rigorous argument, it is necessary to follow an indirect route. 
For $\lambda \in \mathbb{C}$, let $E_{\lambda}$ be the differential operator on $\mathbb{R}^{n}$ defined by

$$
E_{\lambda}=(2 \lambda-n+2) \frac{\partial}{\partial \xi_{n}}+\xi_{n} \Delta
$$

where $\Delta=\sum_{j=1}^{n} \frac{\partial^{2}}{\partial \xi_{j}^{2}}$ is the usual Laplacian on $\mathbb{R}^{n}$. Notice that the operator $E_{\lambda}$ maps $\mathcal{S}\left(\mathbb{R}^{n}\right)$ $\left(\right.$ resp. $\left.\mathcal{S}^{\prime}\left(\mathbb{R}^{n}\right)\right)$ into $\mathcal{S}\left(\mathbb{R}^{n}\right)$ (resp. $\left.\mathcal{S}^{\prime}\left(\mathbb{R}^{n}\right)\right)$, so that we may consider the composition $J_{\lambda+1} \circ E_{\lambda}$.

Lemma 4.7. For $f \in \mathcal{S}\left(\mathbb{R}^{n}\right)$,

$$
\begin{aligned}
& \left(\left(J_{\lambda+1} \circ E_{\lambda}\right) \hat{f} \widehat{)}(\eta)\right. \\
& =-i 2^{-n+2+2 \lambda} \pi^{\frac{n}{2}}\left((\lambda-n+1) h_{n-2 \lambda}(\eta) \frac{\partial \widehat{f}}{\partial \eta_{n}}(\eta)+(2 \lambda-n) \eta_{n} h_{-2 \lambda+n-2}(\eta) \widehat{f}(\eta)\right) .
\end{aligned}
$$

Proof. Using (4.2) and (4.3),

$$
\begin{aligned}
\left(E_{\lambda} f\right)^{\widehat{\gamma}}(\eta) & =(-i)(2 \lambda-n+2) \eta_{n} \widehat{f}(\eta)+(-i) \frac{\partial}{\partial \eta_{n}}\left(-|\eta|^{2} \widehat{f}(\eta)\right) \\
& =(-i)\left((2 \lambda-n) \eta_{n} \widehat{f}(\eta)-|\eta|^{2} \frac{\partial \widehat{f}}{\partial \eta_{n}}(\eta)\right)
\end{aligned}
$$

Next

$$
\begin{aligned}
& \left(\left(J_{\lambda+1} \circ E_{\lambda}\right) f\right)^{\widehat{(}}(\eta)=\widehat{h}_{-2 n+2 \lambda+2}(\eta)\left(E_{\lambda} f\right)^{\widehat{(}}(\eta) \\
& =2^{-n+2+2 \lambda} \pi^{\frac{n}{2}}(-i)\left((2 \lambda-n) \eta_{n} h_{n-2-2 \lambda}(\eta) \widehat{f}(\eta)-(-\lambda+n-1) h_{n-2 \lambda}(\eta) \frac{\partial \widehat{f}}{\partial \eta_{n}}(\eta)\right) .
\end{aligned}
$$

Comparison of (4.5) and (4.7) yields the next result.

\section{Proposition 4.8.}

$$
M \circ J_{\lambda}=\frac{1}{4(\lambda-n+1)} J_{\lambda+1} \circ E_{\lambda} .
$$

Remark 4.9. This equality has to be understood as an equality of operators from $\mathcal{S}\left(\mathbb{R}^{n}\right)$ into $\mathcal{S}^{\prime}\left(\mathbb{R}^{n}\right)$. For $\lambda=n-1, J_{\lambda+1}=J_{n}$ is equal (up to a constant $\neq 0$ ) to the operator $f \longmapsto\left(\int_{\mathbb{R}^{n}} f(\xi) d \xi\right) 1$. Now for $f \in \mathcal{S}\left(\mathbb{R}^{n}\right), \int_{\mathbb{R}^{n}} E_{\lambda} f(\xi) d \xi=0$ as is easily seen by integration by parts. Hence, $J_{\lambda+1} \circ E_{\lambda}$ vanishes for $\lambda=n-1$, so that (4.8) has to be interpreted as a residue formula.

Proposition 4.10. Let $f \in C_{c}^{\infty}\left(\mathbb{R}^{n}\right)$ and assume that $g \in G^{\prime}$ is such that $g^{-1}$ is defined on $\operatorname{Supp}(f)$. Then

$$
\left(E_{\lambda} \circ \rho_{\lambda}(g)\right) f=\left(\rho_{\lambda+1}(g) \circ E_{\lambda}\right) f .
$$

Proof. As a consequence of the intertwining property of $J_{\lambda}$ (Proposition 4.2) and of $M$ (Proposition 3.1),

$$
\left(M \circ J_{\lambda}\right)\left(\rho_{\lambda}(g) f\right)=\rho_{n-\lambda-1}(g)\left(M \circ J_{\lambda}\right) f .
$$

Hence, by (4.8) (assuming for a while that $\lambda \neq n-1$ )

$$
\left(J_{\lambda+1} \circ E_{\lambda}\right) \rho_{\lambda}(g) f=\rho_{n-\lambda-1}(g)\left(\left(J_{\lambda+1} \circ E_{\lambda}\right) f\right) .
$$


Now $\operatorname{Supp}\left(E_{\lambda} f\right) \subset \operatorname{Supp}(f)$, so that $g^{-1}$ is defined on $\operatorname{Supp}\left(E_{\lambda} f\right)$. Hence, by Proposition 4.2

$$
\left(\rho_{n-\lambda-1}(g) \circ J_{\lambda+1}\right) E_{\lambda} f=\left(J_{\lambda+1} \circ \rho_{\lambda+1}(g)\right) E_{\lambda} f
$$

so that

$$
J_{\lambda+1}\left(\left(E_{\lambda} \circ \rho_{\lambda}(g)\right) f\right)=J_{\lambda+1}\left(\left(\rho_{\lambda+1}(g) \circ E_{\lambda}\right) f\right) .
$$

Now, for $\lambda$ generic, the operator $J_{\lambda+1}$ is injective on $\mathcal{S}\left(\mathbb{R}^{n}\right)$, hence

$$
E_{\lambda} \circ \rho_{\lambda}(g) f=\rho_{\lambda+1}(g) \circ E_{\lambda} f .
$$

The general result follows by continuity, as the family $E_{\lambda}$ depends holomorphically on $\lambda$.

Proof of Theorem 3.2. The covariance property of the differential operator $E_{\lambda}$ allows to construct a global differential operator on $S$ which is expressed in the non-compact picture to $E_{\lambda}$. In fact to fully cover the sphere $S$, we only need another chart, which can be chosen as the analog of the map $c$ but constructed from the stereographic projection corresponding to the pole $\mathbf{1}=(1,0, \ldots, 0)$ instead of $\mathbf{- 1}$. Consider the element $s$ of $G$ given by

$$
s=\left(\begin{array}{cccccc}
1 & 0 & 0 & 0 & \ldots & 0 \\
0 & -1 & 0 & 0 & \ldots & 0 \\
0 & 0 & -1 & 0 & \ldots & 0 \\
0 & 0 & 0 & 1 & & 0 \\
\vdots & \vdots & \vdots & & \ddots & \vdots \\
0 & 0 & 0 & 0 & \ldots & 1
\end{array}\right)
$$

Then $s$ acts on $S$ by

$$
s(x)=s\left(\begin{array}{c}
x_{0} \\
x_{1} \\
x_{2} \\
\vdots \\
x_{n}
\end{array}\right)=\left(\begin{array}{c}
-x_{0} \\
-x_{1} \\
x_{2} \\
\vdots \\
x_{n}
\end{array}\right) .
$$

In particular, $s$ maps $\mathbf{- 1}$ to $\mathbf{1}$ and preserves $S^{\prime}$. In the non-compact picture, the map $s$ is defined for $\xi \neq 0$ and is given by

$$
\left(\xi_{1}, \xi_{2}, \ldots, \xi_{n}\right) \longmapsto\left(-\frac{\xi_{1}}{|\xi|^{2}}, \frac{\xi_{2}}{|\xi|^{2}}, \ldots, \frac{\xi_{n}}{|\xi|^{2}}\right) .
$$

The two charts $\xi \longmapsto c(\xi)$ and $\xi \longmapsto s(c(\xi))$ cover $S$. Their common domain corresponds to $\xi \neq 0$, the change of chart being given by (4.9), which is the local expression in the non-compact picture of the transform $s$. So Proposition 4.10, when applied to $g=s$ is exactly what is needed to prove that there is a global differential operator $\mathbf{E}_{\lambda}$ on $S$ which is expressed by $E_{\lambda}$ in the non-compact model. Clearly $\mathbf{E}_{\lambda}$ satisfies

$$
\forall g \in G^{\prime}, \quad \mathbf{E}_{\lambda} \circ \pi_{\lambda}(g)=\pi_{\lambda+1}(g) \circ \mathbf{E}_{\lambda} .
$$

By (4.8),

$$
M \circ I_{\lambda}=\frac{1}{4(\lambda-n+1)} I_{\lambda+1}(g) \circ \mathbf{E}_{\lambda} .
$$


Compose both sides with $I_{n-\lambda-1}$ and use (2.3) to get

$$
\mathbf{D}_{\lambda}=\frac{\pi^{-n}}{4(\lambda-n+1) \Gamma(n-\lambda-1) \Gamma(\lambda+1)} \mathbf{E}_{\lambda}
$$

or equivalently

$$
\widetilde{\mathbf{D}}_{\lambda}=-\frac{1}{4 \pi^{n}} \mathbf{E}_{\lambda}
$$

This relation implies in particular that $\widetilde{\mathbf{D}}_{\lambda}$ is a differential operator on $S$.

\section{The families $\mathrm{D}_{\lambda, N}, \widetilde{\mathrm{D}}_{\lambda, N}$ and $\mathrm{D}_{N}(\lambda)$}

For $N \geq 1$, set

$$
\widetilde{\mathbf{D}}_{\lambda, N}=\widetilde{\mathbf{D}}_{\lambda+N-1} \circ \cdots \circ \widetilde{\mathbf{D}}_{\lambda} \text {. }
$$

Let $M^{N}$ be the operator on $C^{\infty}(S)$ given by multiplication by $x_{n}^{N}$. Set

$$
\mathbb{D}_{\lambda, N}=I_{n-N-\lambda} \circ M^{N} \circ I_{\lambda}
$$

\section{Proposition 5.1.}

i) $\widetilde{\mathbf{D}}_{\lambda, N}$ and $\mathbb{D}_{\lambda, N}$ are differential operators on $S$ which intertwine $\pi_{\lambda \mid G^{\prime}}$ and $\pi_{\lambda+N \mid G^{\prime}}$.

ii)

$$
\widetilde{\mathbf{D}}_{\lambda, N}=\pi^{n(N-1)} \Gamma(\lambda+N) \Gamma(n-\lambda-N) \mathbb{D}_{\lambda, N}
$$

Proof. Repeated uses of (3.2) show that, for any $\mu \in \mathbb{C}, M^{N}$ intertwines $\pi_{\mu \mid G^{\prime}}$ and $\pi_{\mu-N \mid G^{\prime}}$. Hence $\mathbb{D}_{\lambda, N}$ intertwines $\pi_{\lambda \mid G^{\prime}}$ and $\pi_{\lambda+N \mid G^{\prime}}$. On the other hand, repeated uses of (3.3) proves that $\widetilde{\mathbf{D}}_{\lambda, N}$ also intertwines $\pi_{\lambda \mid G^{\prime}}$ and $\pi_{\lambda+N \mid G^{\prime}}$.

Next, $\widetilde{\mathbf{D}}_{\lambda, N}$ as a composition of differential operators on $S$ is a differential operator. So it remains to prove (5.1).

Substitute $\mathbf{D}_{\lambda+j}=I_{n-\lambda-j-1} \circ M \circ I_{\lambda+j}$ for $0 \leq j \leq N-1$ to get

$$
\begin{aligned}
& \mathbf{D}_{\lambda+N-1} \circ \mathbf{D}_{\lambda+N-2} \circ \cdots \circ \mathbf{D}_{\lambda} \\
& \quad=I_{-\lambda+n-N} \circ \cdots \circ I_{-\lambda+n-j-1} \circ M \circ I_{\lambda+j} \circ I_{-\lambda+n-j} \circ M \circ I_{\lambda+j-1} \circ \cdots \circ I_{\lambda},
\end{aligned}
$$

and use (2.3) repeatedly for $\lambda+j$ to obtain

$$
\begin{aligned}
& \mathbf{D}_{\lambda+N-1} \circ \mathbf{D}_{\lambda+N-2} \circ \cdots \circ \mathbf{D}_{\lambda} \\
& \quad=\pi^{n(N-1)}\left(\prod_{j=1}^{N-1} \Gamma(\lambda+j) \Gamma(n-\lambda-j)\right)^{-1} I_{-\lambda+n-N} \circ M^{N} \circ I_{\lambda} .
\end{aligned}
$$

Multiply by the appropriate $\Gamma$ factors coming from (3.4) to get the formula.

The group $G^{\prime}$ acts conformally on $S^{\prime}$. The scalar principal series for $G^{\prime} \simeq \mathrm{SO}_{0}(1, n)$ is defined as follows: for $\mu \in \mathbb{C}$, for $g \in G^{\prime}$ and $f \in C^{\infty}\left(S^{\prime}\right)$,

$$
\pi_{\mu}^{\prime}(g) f(x)=\kappa\left(g^{-1}, x\right)^{\mu} f\left(g^{-1}(x)\right), \quad x \in S^{\prime} .
$$


Let res: $C^{\infty}(S) \longrightarrow C^{\infty}\left(S^{\prime}\right)$ be the restriction map from $S$ to $S^{\prime}$, defined for $f \in C^{\infty}(S)$ by $($ res $f)(x)=f(x), x \in S^{\prime}$. The last remark makes clear that for $\lambda \in \mathbb{C}$ and for $g \in G^{\prime}$,

$$
\operatorname{res} \circ \pi_{\lambda}(g)=\pi_{\lambda}^{\prime}(g) \circ \operatorname{res} .
$$

Define the differential operator $\mathbf{D}_{N}(\lambda): C^{\infty}(S) \longrightarrow C^{\infty}\left(S^{\prime}\right)$ by

$$
\mathbf{D}_{N}(\lambda)=\operatorname{res} \circ \widetilde{\mathbf{D}}_{\lambda, N} .
$$

Theorem 5.2. $\mathbf{D}_{N}(\lambda)$ satisfies

$$
\forall g \in G^{\prime} \quad \mathbf{D}_{N}(\lambda) \circ \pi_{\lambda}(g)=\pi_{\lambda+N}^{\prime}(g) \circ \mathbf{D}_{N}(\lambda) .
$$

The proof follows immediately from the covariance property of $\widetilde{\mathbf{D}}_{\lambda, N}$ and of the restriction map (5.3).

\section{The family $E_{N}(\lambda)$}

The previous constructions of differential operators made for $S$ and $S^{\prime}$ can be made in a similar manner in the non compact picture, i.e., for $\mathbb{R}^{n}$ and $\mathbb{R}^{n-1}$. For $N \in \mathbb{N}$, let $E_{\lambda, N}$ be defined by

$$
E_{\lambda, N}=E_{\lambda+N-1} \circ \cdots \circ E_{\lambda}
$$

and

$$
E_{N}(\lambda)=\operatorname{res} \circ E_{\lambda, N}
$$

where res is the restriction from $\mathbb{R}^{n}$ to $\mathbb{R}^{n-1}$. Then $E_{\lambda, N}$ is a differential operator on $\mathbb{R}^{n}$ which is covariant with respect to $\left(\rho_{\lambda \mid G^{\prime}}, \rho_{\lambda+N \mid G^{\prime}}\right)$ and $E_{N}(\lambda)$ is a differential operator from $\mathbb{R}^{n}$ to $\mathbb{R}^{n-1}$ which is covariant with respect to $\left(\rho_{\lambda \mid G^{\prime}}, \rho_{\lambda+N}^{\prime}\right){ }^{3}$

In this section, for the sake of completeness, we compare $E_{N}(\lambda)$ with Juhl's operator for the non compact model. For $\xi \in \mathbb{R}^{n}$, introduce the notation $\xi=\left(\xi^{\prime}, \xi_{n}\right)$ where $\xi^{\prime} \in \mathbb{R}^{n-1}$. Let $\Delta^{\prime}=\sum_{j=1}^{n-1} \frac{\partial^{2}}{\partial \xi_{j}^{2}}$

Proposition 6.1. Let $E: C^{\infty}\left(\mathbb{R}^{n}\right) \longrightarrow C^{\infty}\left(\mathbb{R}^{n-1}\right)$ be a differential operator and assume that $E$ is covariant with respect to $\left(\rho_{\lambda \mid G^{\prime}}, \rho_{\lambda+N}^{\prime}\right)$ for some $N \in \mathbb{N}$. Then there exits a family of complex constants $a_{j}, 0 \leq j \leq\left[\frac{N}{2}\right]$ such that

$$
E=\operatorname{res} \circ \sum_{j=0}^{\left[\frac{N}{2}\right]} a_{j}\left(\frac{\partial}{\partial \xi_{n}}\right)^{N-2 j} \Delta^{\prime j} .
$$

Proof. By the definition of a differential operator from $\mathbb{R}^{n}$ to $\mathbb{R}^{n-1}, E$ can be written as a locally finite sum

$$
\sum_{i, J} a_{i, J}\left(\xi^{\prime}\right) \operatorname{res} \circ\left(\frac{\partial}{\partial \xi_{n}}\right)^{i} \partial^{J},
$$

where $J=\left(j_{1}, j_{2}, \ldots, j_{n-1}\right)$ is a $(n-1)$-tuple, $\partial^{J}=\prod_{k=1}^{n-1}\left(\frac{\partial}{\partial \xi_{k}}\right)^{j_{k}}$ and $a_{i, J}$ is a smooth function of $\xi^{\prime} \in \mathbb{R}^{n-1}$.

\footnotetext{
${ }^{3}$ The representation $\rho^{\prime}$ is the principal series for $G^{\prime}$ realized in the $\mathbb{R}^{n-1}$, defined in analogy with (5.2).
} 
The invariance by translations forces the $a_{i, J}$ to be constants (and also the sum to be finite). The invariance by $\mathrm{SO}(n-1)$ forces the expression to be of the form

$$
\sum_{i, j} a_{i, j}\left(\frac{\partial}{\partial \xi_{n}}\right)^{i}\left(\Delta^{\prime}\right)^{j}
$$

and finally the covariance under the action of the dilations forces $i+2 j=N$. The statement follows.

Notice that the proof uses only the covariance property for the parabolic subgroup of affine conformal diffeomorphisms of $\mathbb{R}^{n-1}$. The full covariance condition implies further conditions on the coefficients $a_{i, j}$, explicitly written by A. Juhl (see [6], condition (5.1.2) for $N$ even and (5.1.22) for $N$ odd), proving in particular that there exists (up to a constant) a unique covariant differential operator. Now let

$$
E_{N}(\lambda)=\sum_{j=0}^{\left[\frac{N}{2}\right]} a_{j}(\lambda, N)\left(\frac{\partial}{\partial \xi_{n}}\right)^{N-2 j} \Delta^{\prime j}
$$

where $a_{j}(\lambda, N)$ are complex numbers.

To find the ratio between $E_{N}(\lambda)$ and the corresponding Juhl's operator, it is enough to know some coefficient of $E_{N}(\lambda)$ and to compare it to the corresponding coefficient of Juhl's operator. It turns out that the coefficient $a_{0}(\lambda, N)$ is rather easy to compute.

\section{Lemma 6.2.}

i) For $k \in \mathbb{N}$ and $\mu \in \mathbb{C}$,

$$
E_{\mu} \xi_{n}^{k}=k(2 \mu-n+1+k) \xi_{n}^{k-1} .
$$

ii) For $N \in \mathbb{N}$ and for $\lambda \in \mathbb{C}$,

$$
E_{\lambda, N}\left(\xi_{n}^{N}\right)=N !(2 \lambda-n+N+1)(2 \lambda-n+N+2) \cdots(N+2 \lambda-n+2 N) .
$$

iii) The constant $a_{0}(\lambda, N)$ is given by

$$
a_{0}(\lambda, N)=(2 \lambda-n+N+1)(2 \lambda-n+N+2) \cdots(2 \lambda-n+2 N) .
$$

Proof. Let $f$ be a function on $\mathbb{R}^{n}$ which depends only on $\xi_{n}$. Then $\Delta^{\prime} f=0$, and

$$
E_{\mu} f=\left((2 \mu-n+2) \frac{\partial}{\partial \xi_{n}}+\xi_{n} \frac{\partial^{2}}{\partial \xi_{n}^{2}}\right) f
$$

so that $i$ ) and $i$ ) are reduced to elementary one variable computations. For $i i i$ ) observe that

$$
E_{\lambda, N}\left(\xi_{n}^{N}\right)=a_{0}(\lambda, N)\left(\frac{\partial}{\partial \xi_{n}}\right)^{N}\left(\xi_{n}^{N}\right)+0+\cdots+0=N ! a_{0}(\lambda, N)
$$

hence $E_{N}(\lambda)\left(\xi_{n}^{N}\right)=N ! a_{0}(\lambda, N)$ and $\left.i i i\right)$ follows.

The comparison with Juhl's operator is then easy. As his normalization depends on the parity of $N$, one has to examine two cases. 
- In the even case, $E_{N}(\lambda)$ is obtained by multiplying Juhl's operator by

$$
\frac{N !}{\left(\frac{N}{2}\right) !} 2^{\frac{N}{2}-1} \prod_{j=1}^{\frac{N}{2}}(2 \lambda-n+N+2 j) .
$$

- In the odd case, $E_{N}(\lambda)$ is obtained by multiplying Juhl's operator by

$$
\frac{N !}{\left(\frac{N-1}{2}\right) !} 2^{\frac{N+1}{2}} \prod_{j=0}^{\frac{N-1}{2}}(2 \lambda-n+N+1+2 j) .
$$

\section{The operator $\mathrm{D}_{\lambda}$ in the ambient space model}

This last section is devoted to another (simpler) construction of (a multiple of) the operator $\mathbf{D}_{\lambda}$, using the ambient space realization of the principal series.

Let $\Xi^{+}$be the positive light cone,

$$
\Xi^{+}=\{\mathbf{x} \in \mathbf{E}, Q(\mathbf{x})=[\mathbf{x}, \mathbf{x}]=0, t(\mathbf{x})>0\} .
$$

For $\lambda \in \mathbb{C}$, let

$$
\mathcal{H}_{\lambda}=\left\{F \in C^{\infty}\left(\Xi^{+}\right), F(t \mathbf{x})=t^{-\lambda} F(\mathbf{x}), \text { for } t \in \mathbb{R}^{+}\right\}
$$

The space $\mathcal{H}_{\lambda}$ is in one-to-one correspondence with the space $C^{\infty}(S)$ through the map $R_{\lambda}$

$$
\mathcal{H}_{\lambda} \ni F \longmapsto R_{\lambda} F \in C^{\infty}(S), \quad R_{\lambda} F(x)=F((1, x)) .
$$

The space $\mathcal{H}_{\lambda}$ inherits the corresponding topology. For $g \in G$, and $F \in \mathcal{H}_{\lambda}$, let

$$
\Pi_{\lambda}(g) F=F \circ g^{-1} .
$$

Then $\Pi_{\lambda}$ defines a representation of $G$ on $\mathcal{H}_{\lambda}$ and it is easily verified that

$$
R_{\lambda} \circ \Pi_{\lambda}(g)=\pi_{\lambda}(g) \circ R_{\lambda},
$$

so that $\Pi_{\lambda}$ is yet another model for the representation $\pi_{\lambda}$ of $G$.

Let $\square=\frac{\partial^{2}}{\partial t^{2}}-\sum_{j=0}^{n} \frac{\partial^{2}}{\partial x_{j}^{2}}$ be the d'Alembertian on E. It satisfies, for any $g \in G$ and $F$ a smooth function on $\mathbf{E}$

$$
\square(F \circ g)=(\square F) \circ g .
$$

The following lemma, which I learnt from [3] is a key result for what follows.

Lemma 7.1. Let $F_{1}, F_{2}$ be two smooth functions defined in a neighborhood of $\Xi^{+}$, positively homogeneous of degree $-\frac{n}{2}+1$ and which coincide on $\Xi^{+}$. Then $\square F_{1}$ and $\square F_{2}$ coincide on $\Xi^{+}$.

Proof. The function $F_{1}-F_{2}$ vanishes on $\Xi^{+}$. Notice that $d Q(\mathbf{x}) \neq 0$ for any $\mathbf{x} \in \Xi^{+}$. Hence, there exists a smooth function $G$ defined on a neighborhood of $\Xi^{+}$such that

$$
F_{1}(\mathbf{x})-F_{2}(\mathbf{x})=Q(\mathbf{x}) G(\mathbf{x})
$$


Moreover, $G$ is positively homogeneous of degree $-\frac{n}{2}-1$. Now, for any smooth function $H$ on $\mathbf{E}$

$$
\square(Q H)=2(n+2) H+4 E H+Q \square H,
$$

where $E=t \frac{\partial}{\partial t}+\sum_{j=0}^{n} x_{j} \frac{\partial}{\partial x_{j}}$ is the Euler operator. As $G$ is homogeneous of degree $-\frac{n}{2}-1$,

$$
E G(\mathbf{x})=\left(-\frac{n}{2}-1\right) G(\mathbf{x}),
$$

and hence $\square(Q G)(x)=0$ for $x \in \Xi^{+}$. The lemma follows.

The next result is a reformulation of the previous lemma.

Lemma 7.2. Let $F \in \mathcal{H}_{\frac{n}{2}-1}$. Extend $F$ smoothly to a positively homogeneous function of degree $-\frac{n}{2}+1$ to neighborhood of $\Xi^{+}$. Then the restriction to $\Xi^{+}$of $\square F$ does not depend on the extension.

The operator $\square$ induces a map from $\mathcal{H}_{\frac{n}{2}-1}$ to $\mathcal{H}_{\frac{n}{2}+1}$ and intertwines the action of $G$. Let $\Delta_{S}$ be the operator defined on $C^{\infty}(S)$ by

$$
\Delta_{S}=R_{\frac{n}{2}+1} \circ \square \circ R_{\frac{n}{2}-1}^{-1} .
$$

The invariance of $\square$ (see (7.2)) and the covariance of $R_{\lambda}$ (see (7.1)) imply the following proposition.

Proposition 7.3. The operator $\Delta_{S}$ (conformal Laplacian or Yamabe operator on $S$ ) is a differential operator on $S$ which is covariant with respect to $\left(\pi_{\frac{n}{2}-1}, \pi_{\frac{n}{2}+1}\right)$.

Let $\mathbf{B}_{\mu}$ be the differential operator on $\mathbf{E}$ defined by

$$
\mathbf{B}_{\mu} F(\mathbf{x})=x_{n} \square F(\mathbf{x})-2 \mu \frac{\partial F}{\partial x_{n}} .
$$

Lemma 7.4. Let $\mu \in \mathbb{C}$. Let $F$ be a smooth function on $\mathbf{E}$. Then, on $\left\{x_{n} \neq 0\right\}$,

$$
\mathbf{B}_{\mu} F(\mathbf{x})=x_{n}\left|x_{n}\right|^{-\mu} \square\left(\left|x_{n}\right|^{\mu} F\right)(\mathbf{x})+\mu(\mu-1) \frac{1}{x_{n}} F(\mathbf{x}) .
$$

Proof. By an elementary calculation,

$$
\square\left(\left|x_{n}\right|^{\mu} F\right)(\mathbf{x})=\left|x_{n}\right|^{\mu} \square F(\mathbf{x})-2 \mu \operatorname{sgn}\left(x_{n}\right)\left|x_{n}\right|^{\mu-1} \frac{\partial F}{\partial x_{n}}(\mathbf{x})-\mu(\mu-1)\left|x_{n}\right|^{\mu-2} F(\mathbf{x}),
$$

so that

$$
\square\left(\left|x_{n}\right|^{\mu} F\right)+\mu(\mu-1)\left|x_{n}\right|^{\mu-2} F=\operatorname{sgn}\left(x_{n}\right)\left|x_{n}\right|^{\mu-1} \mathbf{B}_{\mu} F .
$$

The conclusion follows, by noticing that $x_{n}=\operatorname{sgn}\left(x_{n}\right)\left|x_{n}\right|$.

Proposition 7.5. Let $g \in G^{\prime}$. Then for $F$ a smooth function on $\mathbf{E}$,

$$
\mathbf{B}_{\mu}(F \circ g)=\left(\mathbf{B}_{\mu} F\right) \circ g .
$$

Proof. As $g \in G^{\prime}$, the coordinate $x_{n}$ is unchanged by the action of $g$, and the action of $g$ commutes with $\frac{\partial}{\partial x_{n}}$ and with $\square$. The result follows. 
Proposition 7.6. Let $F \in \mathcal{H}_{\lambda}$. Extend $F$ smoothly to a neighborhood of $\Xi^{+}$as a positively homogeneous function of degree $-\lambda$. Then the restriction to $\Xi^{+}$of $\mathbf{B}_{\lambda-\frac{n}{2}+1} F$ does not depend on the extension.

Proof. The function $\left|x_{n}\right|^{\lambda-\frac{n}{2}+1} F(\mathbf{x})$ is homogenous of degree $-\frac{n}{2}+1$, and hence, by Lemma 7.2, for $x \in \Xi^{+}, \square\left(\left|x_{n}\right|^{\lambda-\frac{n}{2}+1} F\right)(\mathbf{x})$ only depend on the values of $F$ on $\Xi^{+}$. Hence, by (7.3), for $\mathbf{x}$ in $\Xi^{+}, x_{n} \neq 0, \mathbf{B}_{\lambda-\frac{n}{2}+1} F(\mathbf{x})$ does not depend on the extension of $F$. The result follows by continuity.

Proposition 7.7. The differential operator $\mathbf{B}_{\lambda-\frac{n}{2}+1}$ induces a map from $\mathcal{H}_{\lambda}$ into $\mathcal{H}_{\lambda+1}$, which commutes with the action of $G^{\prime}$.

Proof. The invariance follows from Proposition 7.5.

Having constructed a covariant operator in the ambient space model, it is possible to express it both in the non-compact and in the compact picture.

Proposition 7.8. The local expression of the operator $\mathbf{B}_{\lambda-\frac{n}{2}+1}$ in the non compact picture is equal to $-E_{\lambda}$.

Proof. Let $f$ be a smooth function on $\mathbb{R}^{n}$. Recall the map $c$ (cf. (4.1)) which realizes the passage from $\mathbb{R}^{n}$ to $S$. Its inverse is given by

$$
S \backslash\{\mathbf{- 1}\} \ni\left(x_{0}, x_{1}, \ldots, x_{n}\right) \longmapsto\left(\frac{x_{1}}{1+x_{0}}, \ldots, \frac{x_{n}}{1+x_{0}}\right) .
$$

So map $f$ to a function on $S$ by

$$
C_{\lambda}^{-1} f(x)=\left(1+x_{0}\right)^{-\lambda} f\left(\frac{x_{1}}{1+x_{0}}, \ldots, \frac{x_{n}}{1+x_{0}}\right) .
$$

Consider the function $F$ on $\mathbf{E}$ defined by

$$
F(\mathbf{x})=\left(t+x_{0}\right)^{-\lambda} f\left(\frac{x_{1}}{t+x_{0}}, \ldots, \frac{x_{n}}{t+x_{0}}\right) .
$$

Then $F$ is homogenous of degree $-\lambda$ and coincide on $S$ with $C_{\lambda}^{-1} f$. To compute $\mathbf{B}_{\lambda-\frac{n}{2}+1} F$, first observe that

$$
\frac{\partial F}{\partial t}=\frac{\partial F}{\partial x_{0}}, \quad \frac{\partial^{2} F}{\partial t^{2}}=\frac{\partial^{2} F}{\partial x_{0}^{2}}
$$

so that

$$
\square F=-\sum_{j=1}^{n} \frac{\partial^{2} F}{\partial x_{j}^{2}} .
$$

Hence

$$
\begin{aligned}
\mathbf{B}_{\lambda-\frac{n}{2}+1} F(\mathbf{x})= & -\left(t+x_{0}\right)^{-\lambda-2} x_{n}(\Delta f)\left(\frac{x_{1}}{t+x_{0}}, \ldots, \frac{x_{n}}{t+x_{0}}\right) \\
& -2\left(\lambda-\frac{n}{2}+1\right)\left(t+x_{0}\right)^{-\lambda}\left(t+x_{0}\right)^{-1} \frac{\partial f}{\partial \xi_{n}}\left(\frac{x_{1}}{t+x_{0}}, \ldots, \frac{x_{n}}{t+x_{0}}\right) .
\end{aligned}
$$

Now letting $\mathbf{x}=(1, c(\xi))$,

$$
\mathbf{B}_{\lambda-\frac{n}{2}+1} F(1, c(\xi))=-\xi_{n} \Delta f(\xi)-(2 \lambda-n+2) \frac{\partial f}{\partial \xi_{n}}(\xi) .
$$

A comparison with (4.6) implies the result. 
Proposition 7.9. The expression of the operator $\mathbf{B}_{\lambda-\frac{n}{2}+1}$ on $S$ is given by

$$
x_{n}\left|x_{n}\right|^{-\lambda+\frac{n}{2}-1} \Delta_{S} \circ\left|x_{n}\right|^{\lambda-\frac{n}{2}+1}+\left(\lambda-\frac{n}{2}+1\right)\left(\lambda-\frac{n}{2}\right) \frac{1}{x_{n}} .
$$

The expression, a priori defined on $x_{n} \neq 0$ can be continued continuously to all of $S$.

Proof. Let $f \in C^{\infty}(S)$. Then

$$
F(\mathbf{x})=\left(x_{0}^{2}+\cdots+x_{n}^{2}\right)^{-\lambda} f\left(\frac{x_{0}}{\sqrt{x_{0}^{2}+\cdots+x_{n}^{2}}}, \ldots, \frac{x_{n}}{\sqrt{x_{0}^{2}+\cdots+x_{n}^{2}}}\right)
$$

is a function defined on $\mathbf{E} \backslash\{0\}$ which is positively homogeneous of degree $-\lambda$ and such that for $x \in S$,

$$
F(1, x)=f(x) .
$$

By (7.3) with $\mu=\lambda-\frac{n}{2}+1$ and for $\mathbf{x} \neq 0, x_{n} \neq 0$,

$$
\mathbf{B}_{\lambda-\frac{n}{2}+1} F(\mathbf{x})=x_{n}\left|x_{n}\right|^{-\lambda+\frac{n}{2}+1} \square\left(\left|x_{n}\right|^{\lambda-\frac{n}{2}+1} F\right)(\mathbf{x})+\left(\lambda-\frac{n}{2}+1\right)\left(\lambda-\frac{n}{2}\right) \frac{1}{x_{n}} F(\mathbf{x}) .
$$

The function $\left|x_{n}\right|^{\lambda-\frac{n}{2}+1} F(\mathbf{x})$ is positively homogeneous of degree $-\frac{n}{2}+1$. Thus, by Lemma 7.2 and the definition of the Yamabe operator $\Delta_{S}$, for $x \in S$,

$$
\mathbf{B}_{\lambda-\frac{n}{2}+1} F(1, x)=x_{n}\left|x_{n}\right|^{-\lambda+\frac{n}{2}-1} \Delta_{S}\left(\left|x_{n}\right|^{\lambda-\frac{n}{2}+1} f\right)(x)+\left(\lambda-\frac{n}{2}+1\right)\left(\lambda-\frac{n}{2}\right) \frac{1}{x_{n}} f(x),
$$

from which the statement follows, at least for $x_{n} \neq 0$. As $\mathbf{B}_{\lambda-\frac{n}{2}+1}$ induces a smooth differential operator on $S$, the formula determines the operator on all of $S$ by continuity.

\section{Acknowledgements}

It is a pleasure to thank the anonymous referees for their contributions which helped to improve and reshape the initial version of this article.

\section{References}

[1] Beckmann R., Clerc J.-L., Singular invariant trilinear forms and covariant (bi-)differential operators under the conformal group, J. Funct. Anal. 262 (2012), 4341-4376.

[2] Clerc J.-L., Covariant bi-differential operators on matrix space, Ann. Inst. Fourier (Grenoble), to appear, arXiv:1601.07016.

[3] Eelbode D., Souček V., Conformally invariant powers of the Dirac operator in Clifford analysis, Math. Methods Appl. Sci. 33 (2010), 1558-1570.

[4] Fischmann M., Juhl A., Somberg P., Conformal symmetry breaking differential operators on differential forms, arXiv:1605.04517.

[5] Gel'fand I.M., Shilov G.E., Generalized functions. Vol. I: Properties and operations, Academic Press, New York - London, 1964

[6] Juhl A., Families of conformally covariant differential operators, Q-curvature and holography, Progress in Mathematics, Vol. 275, Birkhäuser Verlag, Basel, 2009.

[7] Knapp A.W., Representation theory of semisimple groups. An overview based on examples, Princeton Mathematical Series, Vol. 36, Princeton University Press, Princeton, NJ, 1986.

[8] Kobayashi T., Kubo T., Pevzner M., Conformal symmetry breaking operators for differential forms on spheres, Lecture Notes in Math., Vol. 2170, Springer, Singapore, 2016. 
[9] Kobayashi T., Pevzner M., Differential symmetry breaking operators: I. General theory and F-method, Selecta Math. (N.S.) 22 (2016), 801-845, arXiv:1301.2111.

[10] Kobayashi T., Pevzner M., Differential symmetry breaking operators: II. Rankin-Cohen operators for symmetric pairs, Selecta Math. (N.S.) 22 (2016), 847-911, arXiv:1301.2111.

[11] Kobayashi T., Speh B., Symmetry breaking for representations of rank one orthogonal groups, Mem. Amer. Math. Soc. 238 (2015), v+110 pages, arXiv:1310.3213.

[12] Olver P.J., Classical invariant theory, London Mathematical Society Student Texts, Vol. 44, Cambridge University Press, Cambridge, 1999. 